\title{
The latest BROND-3 developments
}

\author{
A.V. Ignatyuk ${ }^{\mathrm{a}}$ and B.I. Fursov \\ Institute of Physics and Power Engineering, 249033 Obninsk, Russia
}

\begin{abstract}
The last modifications of the general purpose BROND-3 library are reviewed. Main differences from previous evaluations for the structural materials, the advanced coolants, the minor actinides and the most important fission products are discussed. Needs on estimation of evaluated data uncertainties are indicated.
\end{abstract}

\section{Introduction}

The group-constant system ABBN-93, based mainly on the evaluated data library BROND-2 and the compilation FOND2 of selected foreign evaluations, is still used widely in Russia for practical calculations of nuclear reactors. The ABBN-93 data have been carefully validated against many national and international benchmarks and for the most important fuel and structural materials they satisfy the main requests of current reactor-design accuracies. However, for advanced reactors with much higher safety criteria, with an essentially increased operating cycle, and a new fuel and coolant composition the available group-constants cannot guarantee with confidence the required accuracies.

New technologies related to a higher burn-up and a nuclear fuel recycle, a transmutation of minor actinides and longlived fission products require essentially more accurate data for most nuclides, which should be provided with the future updated libraries. In the present report the main works directed on a formation of updated version of the Russian evaluated data library are briefly discussed.

\section{Cross section evaluations for actinides}

The ENDF/B-VII evaluations for main fissile isotopes ${ }^{235} \mathrm{U}$, ${ }^{238} \mathrm{U}$ and ${ }^{239} \mathrm{Pu}$ were improved essentially in the last year for both the resolved resonances and for fast neutrons [1]. The improvements for the resonance region are achieved mainly due to the analysis of complete set of experimental data with the SAMMY (ORNL) code [2], while the improvements for fast neutrons are mainly related to the revision of the neutron standard cross sections [3]. Taking into account an important role of the obtained improvements they were included also in the updated Russian files. However, only the resonance parameters were adopted into BROND-3 without any

${ }^{a}$ Presenting author, e-mail: ignatyuk@ippe.ru corrections, while the cross sections above the resonance region were obtained slightly different due to other approximations of experimental data.

In the last year, the revision of BROND-3 files for ${ }^{232} \mathrm{Th}$ and ${ }^{233} \mathrm{U}$ was completed too [4]. The neutron resonance parameters were taken from the ORNL evaluations, which are included also in ENDF/B-VII [5], but the analysis of all experimental data above the resonance region was performed independently and the evaluated cross sections differ something as from the ENDF/B-VII data, as well from the recent Minsk evaluations [6]. It should be noted that the upper neutron energy in the BROND-3 files for the most actinides is taken at $150 \mathrm{MeV}$ [7]. For the isotopes ${ }^{231} \mathrm{~Pa},{ }^{233} \mathrm{~Pa}$ and ${ }^{234} \mathrm{U}$, which are important for the thorium fuel cycle, the Minsk evaluations [8] were adopted for BROND-3 and the works are caring out now to extend the upper neutron energy for these isotopes too.

The BROND-3 files for minor actinides have been created in 1996-1998. New experimental data on the neutron total and fission cross sections, as well as the delayed neutron yields were obtained during the last years. These data were taken into account in the updated evaluations performed in 2002-2005. For all minor actinides the updated files include evaluations of the integral yields and spectra of gamma-rays, which are absent, as a rule, in the most files included in the recent versions of JENDL-3.3, ENDF/B-VII, and Minsk libraries. There are no essential contradictions between various evaluations for the neutron resonance parameters and the capture cross sections averaged over the fast reactor spectrum, but for the inelastic scattering, fission and threshold reactions the discrepancies between evaluations remain much larger than the required accuracies of the data.

A comparison of the evaluated total, inelastic scattering and fission cross sections for ${ }^{238} \mathrm{Pu}$, shown in figure 1 , can be considered as an example of the existing disagreements. Because this isotope is accumulated under a multiple recycling of nuclear fuel, a more accurate estimation of the inelastic scattering and fission cross sections for ${ }^{238} \mathrm{Pu}$ is a high priority task for the spent-fuel transmutation technology. For the intensive transmutation scenario the requests on data accuracies for this isotope could be as high as the modern requests on the data for ${ }^{238} \mathrm{U}$ and ${ }^{240} \mathrm{Pu}$. 



Fig. 1. Recent evaluations of the neutron total (top), the inelastic scattering (middle), and the fission (bottom) cross sections for ${ }^{238} \mathrm{Pu}$ at energies above $10 \mathrm{keV}$.

\section{Evaluations for structural materials and advanced coolants}

The data files for main structural materials were updated essentially in all libraries at the end of the nineties on the basis of the precise data for the total cross sections obtained on the neutron spectrometers of Geel and Los Alamos $[9,10]$. For isotopes of the iron group the main evaluation problems relate to the observed gross-structure of neutron cross sections in the region of unresolved resonances. It is important to take into account these structures not only for the total cross sections, but for the neutron inelastic scattering too. The recent evaluations of the inelastic scattering cross sections are shown in figure 2. The JEFF-3.1 evaluation is driven directly over the experimental data measured with a high resolution [9] and the

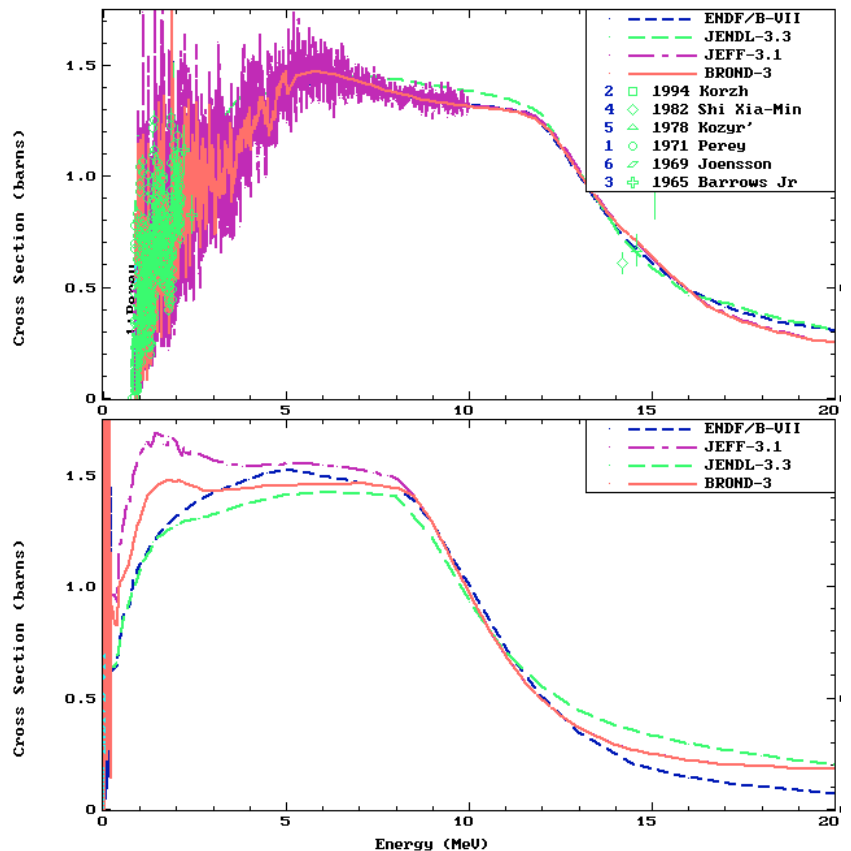

Fig. 2. The neutron inelastic scattering cross sections for ${ }^{56} \mathrm{Fe}$ (top), and ${ }^{57} \mathrm{Fe}$ (bottom).

Table 1. The neutron total and inelastic scattering cross sections averaged over the standard fission-neutron spectrum for two iron isotopes.

\begin{tabular}{lllll}
\hline \multirow{2}{*}{ Library } & \multicolumn{2}{c}{ Fe-56 } & \multicolumn{2}{c}{ Fe-57 } \\
\cline { 2 - 5 } & total & inelastic & total & inelastic \\
\hline JENDL-3.3 & 3.252 & 0.653 & 3.910 & 1.173 \\
\hline JEFF-3.1 & 3.247 & 0.617 & 4.137 & 1.501 \\
\hline ENDF/B-VII & 3.219 & 0.643 & 3.479 & 1.203 \\
\hline BROND-3 & 3.236 & 0.617 & 3.949 & 1.320 \\
\hline
\end{tabular}

cross section fluctuations are conserved in it up to $10 \mathrm{MeV}$. Other evaluations contain the cross sections averaged over the energy interval of $20-50 \mathrm{keV}$ that reduced essentially the cross section fluctuations. Particularly, for the JENDL-3.3 and ENDF/B-VII evaluations such fluctuations disappear already above $3 \mathrm{MeV}$.

However, if we compare the cross sections averaged over wide energy intervals (for example, 28-group constants), all evaluations of the total, inelastic scattering and capture cross sections for ${ }^{56} \mathrm{Fe}$ agree quite well beside the capture cross section above $1 \mathrm{MeV}$ that is small enough and not important for most applications.

The divergence of inelastic scattering evaluations for ${ }^{57} \mathrm{Fe}$ is more serious (fig. 2). It reflects both a very limited amount of experimental data and high uncertainties of the models calculations near the threshold energies. A large difference between evaluations can be displayed clearly comparing the cross sections averaged over the fission-neutron spectrum. Such data for two iron isotopes are given in table 1 .

The differences between the averaged cross sections could be considered as an objective estimation of existing uncertainties of the corresponding data. For ${ }^{56} \mathrm{Fe}$ such uncertainties are rather small: about $1 \%$ for the total cross section and 




Fig. 3. Evaluations of the inelastic scattering cross sections for ${ }^{93} \mathrm{Zr}$ (top) and ${ }^{98}$ Mo (bottom). The JENDL-3.3 evaluations adopted in ENDF/B-VII for both isotopes and in JEFF-3.1 for ${ }^{98}$ Mo.

$6 \%$ for the inelastic scattering, but they are much larger for ${ }^{57} \mathrm{Fe}$ : about $15 \%$ for the total one and $28 \%$ for the inelastic scattering. In spite of a small abundance, ${ }^{57} \mathrm{Fe}$ makes a dominant contribution to the inelastic scattering matrix of natural iron at energies below $1 \mathrm{MeV}$. So, the above uncertainties demonstrate, in fact, the realistic uncertainty of calculations connected with the inelastic scattering effects on of reactor structural materials. Attempts to reduce this uncertainty on the basis of integral benchmarks were mainly unsuccessful and additional experimental data are needed to remove the divergence of evaluations.

Zirconium and molybdenum are important structural materials of the reactor fuel rods. In spite of many experimental data the differences between evaluations of the inelastic scattering and threshold reaction cross sections for isotopes of these elements are unreasonably large. To improve the previous BROND-2 evaluations we reanalyzed the available experimental data on the basis of more consistent theoretical models. A special attention was paid to the adjustment of the optical model parameters to the available data on the neutron total cross sections including the recent precise measurements for natural elements [11]. The new BROND-3 evaluations are close enough to the previous ones for the total and neutron capture cross sections, but differ essentially for the inelastic scattering and $(\mathrm{n}, 2 \mathrm{n})$ cross sections. The evaluations for ${ }^{93} \mathrm{Zr}$ shown in figure 3 can be considered as a typical example of the existing discrepancies. It is obvious nowadays that a contribution of the direct and preequilibrium processes was not taken into account in the JENDL-3.3 and JEFF-3.1 evaluations for the neutron energies above $8 \mathrm{MeV}$. An immediate consequence of that are low values of the inelastic scattering cross section and the overestimated (n,2n) cross section. On the other hand, the underestimation of inelastic scattering in JENDL3.3 at the

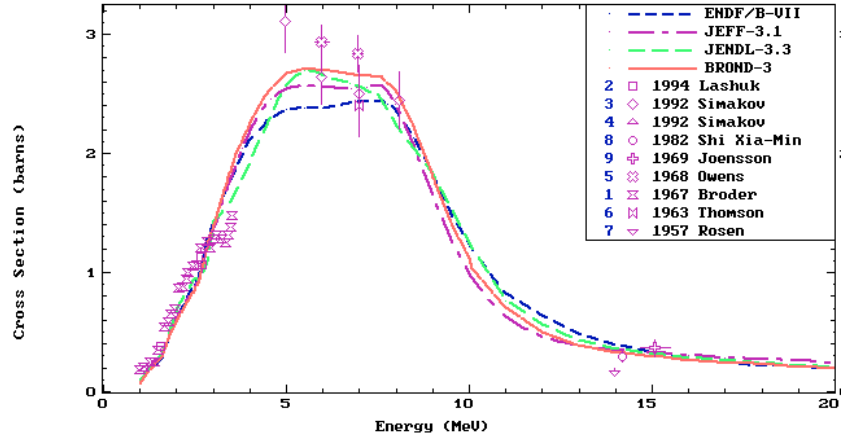

Fig. 4. Experimental data on the inelastic scattering cross sections for ${ }^{209} \mathrm{Bi}$ in comparison with the recent evaluations.

neutron energies below $7 \mathrm{MeV}$ can be related to an adoption of too high value of the imaginary optical potential in the corresponding calculations.

For even-even isotopes of zirconium and molybdenum the direct processes were taken into consideration by the JENDL-3.3 evaluations and, as a result, there are no essential contradictions between the inelastic scattering evaluations at energies above $8-10 \mathrm{MeV}$, but the disagreements at energies below $7 \mathrm{MeV}$ remain approximately the same as for odd isotopes (see fig. 3 for ${ }^{98} \mathrm{Mo}$ ). There are not enough experimental data on the inelastic scattering cross sections to test the available evaluations. By reason of that, only some phenomenological systematics based on the data for neighboring nuclei can be mainly used for the reasonable selection between evaluations. The latest CENDL-3 and BROND-3 evaluations look preferable from this point of view over the previous JENDL-3.3 and JEFF-3.1.

Development of the advanced fast reactors and the intensive neutron sources with a heavy-metal liquid coolant requires an essential increase of evaluated data accuracies for lead and bismuth isotopes. The resonance structure in these isotopes is important up to energies $1.5-2.0 \mathrm{MeV}$ and the parameters of resolved or unresolved resonances should be estimated accurately enough for the entire region. For BROND-3 the previous evaluations of resonance parameters were revised on the basis of the recent data obtained at the GELINA neutron spectrometer [12]. As a result, a large amount of p-wave resonances was added to the previous data. To estimate the optical model parameters and the corresponding absorption cross sections the data on the total and elastic cross sections were carefully analyzed for natural lead and its isotopes. In general, the new BROND-3 results are close enough to the latest JEFF-3.1 evaluations for most cross sections, but some differences exist, nevertheless, for the inelastic scattering and the threshold reactions. As an example, the inelastic scattering evaluations for ${ }^{209} \mathrm{Bi}$ are compared in figure 4 with the corresponding experimental data. In this case we have a rather good agreement between evaluations at the whole energy region, and the ENDF/B-VII evaluation looks only a little low between 4 and $8 \mathrm{MeV}$.

\section{Evaluations for fission products}

Intercomparison of the cross section evaluations for fission products, performed by the WPEC at the nineties [13], 
demonstrated a good enough agreement for the capture cross sections averaged over the fast-reactor neutron spectrum, but a rather large discrepancy for similar data on the inelastic scattering and the $(n, 2 n)$ reactions. The existing disagreements are connected with a very limited amount of experimental data, on the one hand, and using too simplified models in many previous evaluations, on the other hand.

In the last years the analysis of all data libraries for fission products was performed by the WPEC subgroups 21 and by subgroup 23 were worked out the recommendations on the preferable evaluations and possible improvements of previous ones [14]. On the basis of these recommendations and the updated atlas of neutron resonance parameters [15] the new ENDF/B-VII files were assembled for more than 210 fission products. For about 30 nuclides the previous evaluations were recognized as too primitive and they were replaced by completely new ones based on the modern theoretical models. Of course, we are going to adoptee most of the new evaluations for the BROND-3 library too.

In the list of fission products about 40 nuclides can be related to the most important products [13], because they determine in a fast reactor up to $95 \%$ of the total neutron absorption by all fission products. The requirements to the data accuracy for these nuclides are usually much higher than for remaining secondary products, a summary contribution of which to the neutron absorption is less than $5 \%$. The improvements recommended by the Russian members of WPEC subgroups for some of the important products have not been adopted in the ENDF/B-VII files. For such nuclides it was decided to prepare the independent updated BROND-3 evaluations. It should be also noted, that the gamma-ray yields are not included still in many of the ENDF/B-VII files. We would like to add the gamma-ray evaluations for the most important fission products at least.

An example of such fission products is ${ }^{93} \mathrm{Zr}$ that was discussed above and for which the JENDL-3.3 evaluation adopted in ENDF/B-VII cannot be considered as the preferable one. Another example is ${ }^{145} \mathrm{Nd}$, the basic neutron cross sections of which are shown in figure 5. For this nucleus the completely new evaluations were performed for ENDF/BVII, which differ essentially from the previous JENDL-3.3 and JEFF-3.1 for the inelastic scattering and (n,2n) cross sections. Nevertheless, the BROND-3 evaluations for ${ }^{145} \mathrm{Nd}$ look preferable, because they agree better with systematics of the corresponding cross sections for neighboring nuclei. Similar BROND-3 evaluations, different from ENDF/B-VII, have been performed for about 20 nuclides from the list of the most important fission products.

\section{BROND-3 and ROSFOND-2006}

Nowadays the general purpose BROND-3 library includes about 120 nuclides, evaluations of which differ from other libraries. Almost the same list of nuclides was included in the previous BROND-2 library [16]. For practical applications and for the new group-constant preparation, in particularly, other data libraries are used widely for nuclides unavailable in BROND-3. The corresponding files are selected usually on the

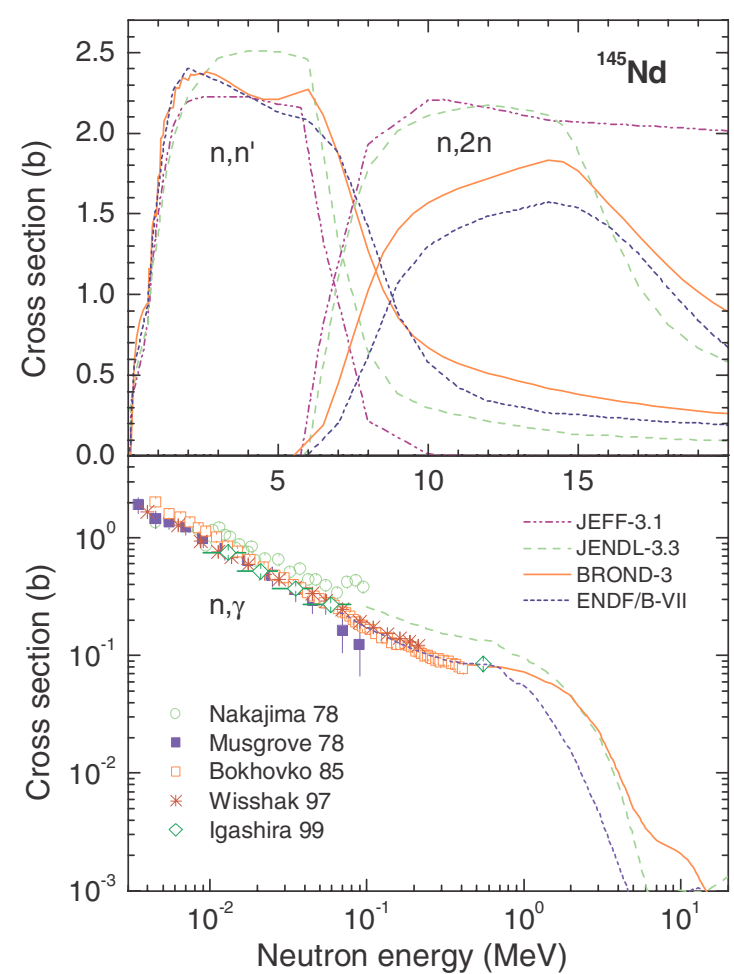

Fig. 5. Evaluations of the inelastic scattering, $(n, 2 n)$ reaction and radiative capture cross sections for ${ }^{145} \mathrm{Nd}$ in comparison with experimental data.

basis of a consistent description of some integral experiments and, first of all, the well-known criticality benchmarks [17].

A new version of such compiled library ROSFOND-2006 was assembled at the IPPE in the last year. The library contains more than 600 nuclides used in various reactor projects [18]. About two thirds of them are the complete general purpose files and a third presents the activation files needed for the analysis of residual activations of reactor material.

The ROSFOND-2006 files are tested now on some selected set on the national and international benchmarks and the obtained results will be used for further updating the BROND-3 files and a subsequent revision of files included in the ROSFOND library.

\section{Uncertainties of evaluated data}

An important task of all libraries still remains a reasonable estimation of evaluated data uncertainties, which are needed for calculation of the resulting integral uncertainties of main reactor characteristics. Uncertainties, based mainly on the expert estimation, were adopted in the ENDF/B-V library, but they were rejected in the following ENDF/B-VI version. Similar expert estimations of the data uncertainties are available for the basic group constants included in the ABBN-93 system, but it is rather difficultly to transform them to the uncertainties of differential data in the general purpose files.

For 19 most important reactor materials the covariance matrices of uncertainties were evaluated for the JENDL-3.3 library [19]. In the last year a similar work has been began for the ENDF/B-VII files, however, the obtained results are not included yet in the library version distributed to users [1]. 


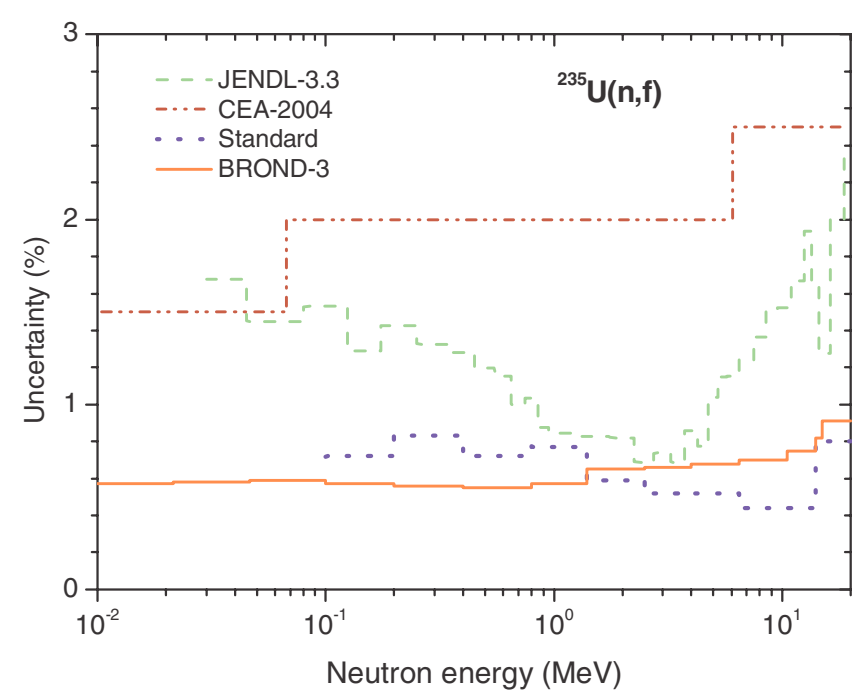

Fig. 6. Relative uncertainties of the fission cross section evaluations for ${ }^{235} \mathrm{U}$ adopted in different libraries.

The analysis of uncertainties for the BROND-3 evaluations is carrying out now on the basis of the unrecognized error estimation method [20]. Beside a consistent consideration of statistical errors of experimental data the method allows to determine some systematic data uncertainties usually underestimated by many authors and to establish also some implicit correlations of data. This approach was used successfully for the independent evaluations of the standard neutron cross sections [3] and is applying nowadays routinely to construct the uncertainties and corresponding covariance matrices of the main fissile nuclei and reactor structural materials for the BROND-3 library.

As an example the uncertainties of the fission cross sections for ${ }^{235} \mathrm{U}$ are compared in figure 6 for different libraries. Rather large discrepancies between the evaluated uncertainties can be clearly seen and a divergence of the covariances, which characterize long-range correlations of the cross sections, is quite large too. Most of contradictions between evaluated uncertainties can be related to underestimation of the systematic errors of experimental data and we hope to obtain a reasonable estimation of such errors in the framework of the developed approach [20].

\section{Conclusion}

Some typical examples of the new BROND-3 evaluations are presented above and their main differences from similar evaluations of other libraries are briefly discussed. Main part of new evaluations relates to the most important fissile and reactor structural materials, while for materials of lower various practical applications. The corresponding recommendations are prepared by the Russian Nuclear Data Working Group and the main part of the selected files is included in the ROSFOND library [18] compiled in the last year. A complete version of the BROND-3 library should be released at the end of 2008 .

\section{References}

1. M.B. Chadwick et al., Nuclear Data Sheets 107, 2931 (2001).

2. L.C. Leal et al., Report ORNL/TM-2000/372 (2001).

3. V.G. Pronyaev et al., Nuclear Data for Science and Technology (Santa Fe, 2004). Eds. M. Chadwick et al. (AIP Conf. Proc. 769, Melville, NY, 2005), p. 808.

4. A.V. Ignatyuk et al., Report INDS (NDS)-494 (IAEA, Vienna, 2006), p. 9.

5. L.C. Leal, H. Derrien, Report INDS (NDS)-468 (IAEA, Vienna, 2004), p. 31.

6. V.M. Maslov et al., Report IAEA NDS (BLR)-018 \& -019 (IAEA, Vienna, 2003).

7. A.V. Ignatyuk et al., Nuclear Data for Science and Technology (Santa Fe, 2004). Eds. M. Chadwick et al. (AIP Conf. Proc. 769, Melville, NY, 2005), p. 103.

8. V.M. Maslov et al., Report IAEA NDS (BLR)-017, -019 \& -020 (IAEA, Vienna, 2004).

9. E. Cornelis et al., EXFOR 22316 (1995).

10. W.P. Abfalterer et al., Phys. Rev. C 63, 044608 (2001).

11. A.V. Ignatyuk et al., VANT, Ser. Nuclear Constants, 2002, Issues $1-2$, p. 3.

12. P. Mutti, DS Thesis, Gent Univ., 1997.

13. H. Gruppelaar et al., Report NEA/WPEC-17 (ECN-R-98-014), OECD, 1998.

14. P. Oblozinsky et al., Nuclear Data for Science and Technology (Santa Fe, 2004). Eds. M. Chadwick et al. (AIP Conf. Proc. 769, Melville, NY, 2005), p. 438.

15. S. Mughabghab, Atlas of Neutron Resonance Parameters (Academic Press, NY, 2006).

16. A.I. Blokhin et al., Nuclear Data for Science and Technology (Gatlinburg, 1994), Ed. J. Dickens (Oak Ridge, 1994), Vol. 2, p. 695.

17. International Handbook of Evaluated Criticality Safety Benchmark Experiments (NEA, Paris, 2005).

18. N.N. Nikolaev, V. Mire Nauki, 2005, Issue 9, p. 103.

19. K.Shibata et al., Nuclear Data for Science and Technology (Tsukuba, 2001), Ed. K. Shibata (JAERI, Tsukuba, 2002), Vol. 1, p. 40.

20. E.V. Gai, Report INDC(NDS)-453 (IAEA, Vienna, 2004), p. 359 . 